\title{
Pott's Spine with Tubercular Meningitis and Primary Optic Atrophy: An Enigma with a Rare Cautionary Tale
}

\author{
Khursheed Alam Khan ${ }^{1} \quad$ Kunj Bihari Saraswat ${ }^{1} \quad$ Tushar Marbate $^{1} \quad$ Ashok Gupta $^{1}$ \\ ${ }^{1}$ Department of Neurosurgery, Sawai Maan Singh Medical College, \\ Address for correspondence Khursheed Alam Khan, MS, \\ Jaipur, Rajasthan, India \\ MCh, 14/17, Kanchana Bihari Marg, Opposite Rajrani Medical \\ Store, Kalyanpur, Lucknow 226022, Uttar Pradesh, India \\ (e-mail: kkneuro15@gmail.com).
}

Indian J Neurosurg 2019;8:199-202

\begin{abstract}
Tuberculosis of spine, known as Pott's spine, is a significant health risk. Misdiagnosis or delayed diagnosis with lack of timely interventions lead to serious neurological complications and is associated with morbidity and mortality. We present a case of Pott's spine who developed tubercular meningitis with decreased vision due to primary optic atrophy, to highlight the significance of thorough clinical and neuroradiological workup with instillation of prompt antitubercular therapy in patients of central nervous

Keywords

- Pott's spine

- tubercular meningitis

- primary optic atrophy system tuberculosis (CNS TB). This association of Pott's spine with decreased vision secondary to primary optic atrophy due to tubercular involvement of the second cranial nerve is very rare. Here, associated risk factors, varied clinical presentations, complications, and treatment of CNS TB are reviewed.
\end{abstract}

\section{Introduction}

Central nervous system tuberculosis accounts for approximately $10 \%$ of extrapulmonary tuberculosis cases and $1 \%$ of all TB cases. ${ }^{1}$ Here, we report a very rare case of Pott's spine with involvement of multiple cranial nerves including second cranial nerve leading to decreased vision. This association of Pott's spine with decreased vision secondary to primary optic atrophy due to tubercular involvement of second cranial nerve $(\mathrm{CN})$ and not secondary to papilledema is very rare and to best of our knowledge only a single case has been reported in literature till now. ${ }^{2}$

\section{Case Report}

A 25-year-old woman presented with sudden-onsent bilateral paraparesis since 3 days. She had mild backache since 8 months which aggravated a month before and she took analgesics herself and neglected the increase in pain. On examination patient was conscious and oriented. Power in both lower limbs was 2/5. Erythrocyte sedimentation rate (ESR) was raised, magnetic resonance imaging (MRI) spine

received

October 16, 2018

accepted

January 28, 2019

published online

November 21, 2019 showed erosion of adjacent endplates of D10 and D11 vertebral bodies with cord compression at this level and erosion of anterior surfaces of D5 to L1 vertebral bodies with pre- and paravertebral abscess at these levels ( - Fig. 1A).

Antitubercular (ATT) 4-drug therapy was started and patient was operated for Pott's spine. D10 and D11 laminectomy with aspiration of pus and spinal fixation was done with no iatrogenic dural tear during the procedure. Histopathology confirmed tuberculosis. Patient's weakness improved to $3 / 5$. On the seventh postoperative day, she developed severe headache and decreased vision. Examination revealed nuchal rigidity, right 3rd and 6th cranial nerves $(\mathrm{CN})$ palsies with bilateral decreased vision. Perception of light was positive in the right eye, and finger counting was present through left at a distance of one foot. Both pupils were mid-dilated and sluggishly reacting. Fundus examination revealed chalky white optic disc on right and pale white optic disc on left side with distinct disc margins. There was diffuse arteriolar attenuation with no evidence of any choroid tubercle. Fundus findings were suggestive of bilateral primary optic atrophy with no features of papilledema ( - Fig. 1C). Computed tomography (CT) scan of brain showed mild dilatation of ventricles and
DOI https://doi.org/

$10.1055 / \mathrm{s}-0039-3400345$

ISSN 2277-954X.
License terms

()(1) $\odot \circledast$ 


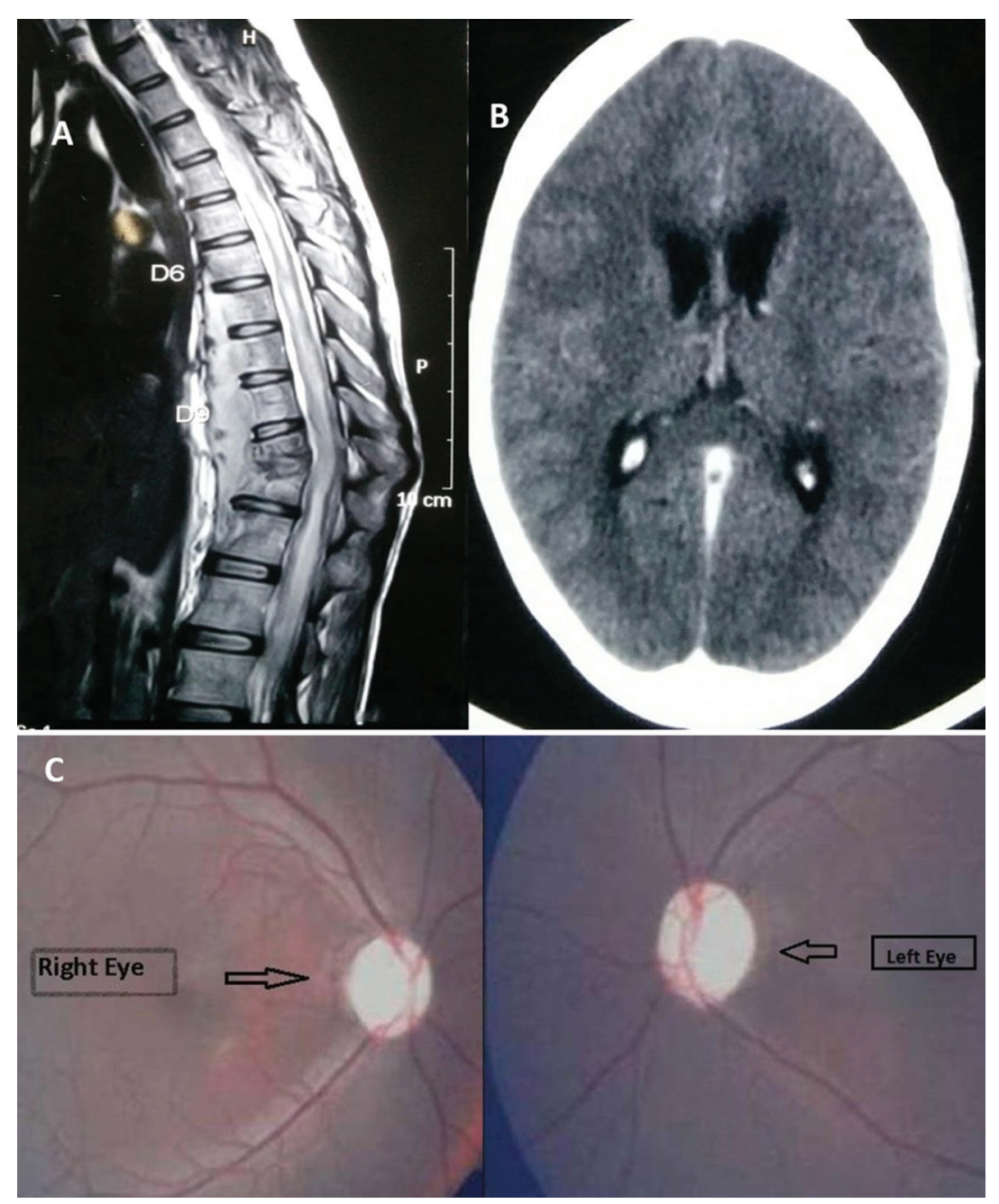

Fig. 1 (A) MRI spine showing erosion of adjacent endplates of D10 and D11 vertebral bodies with cord compression and myelopathic changes at this level along with erosion of anterior surfaces of D5 to L1 vertebral bodies with pre- and paravertebral abscess at these levels. (B) Contrast CT brain showing meningeal and ependymal enhancement with mild dilatation of ventricles. (C) Fundus findings suggestive of bilateral primary optic atrophy with no features of papilledema.

enhancement of basal cisterns ( - Fig. 1B). TBM was suspected and guarded lumbar puncture was done. CSF picture was suggestive of tuberculosis and AFB staining was positive. Steroids were added in the treatment. Her headache relieved and power in both lower limbs improved, but right 3rd and 6th $\mathrm{CN}$ palsies and vision remained same. After 6 weeks follow-up she was relieved of backache and headache. Her power improved in both lower limbs to $4 / 5$, but unfortunately her right 3 rd and 6 th $\mathrm{CN}$ palsies and bilateral decreased vision persisted. Contrast MRI brain at 6 weeks follow-up revealed mild enhancement of optic chiasm and leptomeninges ( - Fig. 2 ).

\section{Discussion}

Pott's spine accounts for approximately $50 \%$ of extrapulmonary TB that involves bones and joints, but has a reported incidence of less than $1 \%$ to $5 \%$ of all TB cases. ${ }^{2} \mathrm{~TB}$ of vertebral body with cord impingement accounts for maximum number of cases with spinal involvement which mostly present with pain, gibbus, and signs of extrinsic cord compression.
Diagnosis of Pott's spine is a big challenge and misdiagnosis is quite common because in early phases the clinical and radiological features are nonspecific and can be confused with malignancy or pyogenic spine involvement on imaging. Diagnosis can, therefore, be delayed for months to years, increasing the potential for neurologic complications. Hence, keen observation and high index of suspicion is required to prevent delay in diagnosis.

Neurologic deficits are noted in $50 \%$ of patients, namely in the form of bowel/bladder incontinence, sensory disturbance, and weakness including paraplegia. ${ }^{2}$ Other rare complications are tubercular meningitis and hydrocephalus, and a very rare complication is involvement of cranial nerves as was seen in our case.

Erythrocyte sedimentation rate (ESR) is a helpful indication toward the disease, with an increase $(>20 \mathrm{~mm} / \mathrm{h})$ to occur in $88 \%$ to $100 \%$ of patients. ${ }^{3} \mathrm{X}$-ray and CT spine are informative, but MRI spine is the modality of choice which can detect early changes in endplates. CT guided or open biopsy later can provide definitive diagnosis. 


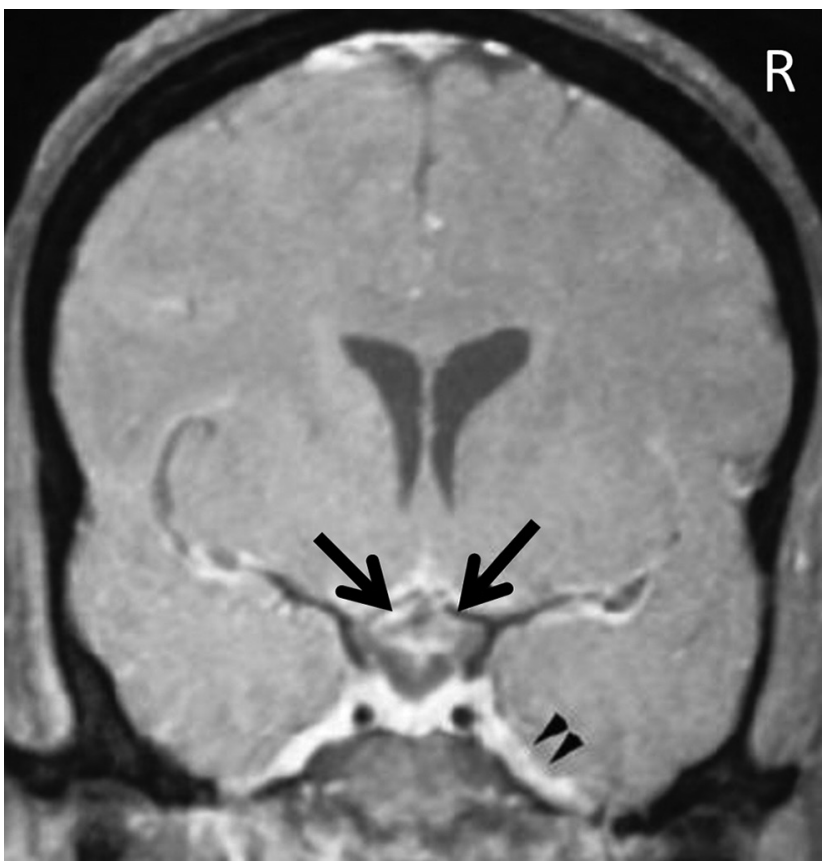

Fig. 2 Contrast MRI brain at 6 weeks follow-up, coronal view, showing mild enhancement of optic chiasm (bilateral arrows) and leptomeninges (arrow heads).

Frequency of TBM is lesser than Pott's spine, comprising $15 \%$ of extrapulmonary TB and $0.7 \%$ of all TB cases $^{4}$. Symptoms appear gradually which progress to meningitic phase within 2 to 3 weeks. CN involvement occurs in 20\% to $30 \%$ cases and most commonly there is involvement of $\mathrm{CN} 6$ and less frequently $\mathrm{CN} 3$ followed by $\mathrm{CN} 4$ and 7. Other $\mathrm{CNs}$ are rarely affected. Involvement of $\mathrm{CN} 2$ is rare and leads to optic atrophy and blindness ${ }^{2}$. This rare involvement of $\mathrm{CN}$ 2 is mostly in the form papilledema leading to secondary optic atrophy as a result of TBM or hydrocephalus, but very rarely primary optic atrophy has also been seen. This can be attributed to the basal exudates in TBM leading to opticochiasmatic arachnoiditis and tubercular vasculitis which ultimately occlude the blood vessels supplying the optic nerve. This ischemic involvement of the optic nerve causes destruction of optic nerve fibers leading to primary optic atrophy. Such rare involvement of second $\mathrm{CN}$ presenting as primary optic trophy was seen in our case. Postoperative visual loss (POVL) could also be suspected as a cause of visual loss secondary to a spinal procedure. But the condition was ruled out because in POVL symptoms of vision loss or field defect are mostly noted in $81 \%$ of the cases by the second postoperative day. ${ }^{5}$ Also, there were no splinter hemorrhages, cherry red spots, venous congestion, etc. on fundus evaluation. These features are commonly found in POVL. ${ }^{5}$ Also it was associated with involvement of other cranial nerves.

Possibility of ethambutol toxicity and paradoxical reaction to ATT were ruled out. Ethambutol toxicity can also present as blindness and other $\mathrm{CN}$ palsies but the possibility was kept to minimum because such a severe bilateral involvement of vision is very rare at the dose of $15 \mathrm{mg} / \mathrm{kg} /$ day. Reported incidence is less than one percent with ethambutol at dose of $15 \mathrm{mg} / \mathrm{kg} / \mathrm{day}$ for two months. ${ }^{6}$ There were no associated risk factors like co association with human immunodeficiency virus (HIV) infection, impairment of renal function, higher dose, diabetes, prolonged duration, etc. Ethambutol toxicity with vision impairment mostly presents between 4 and 12 months after initiating the drug. ${ }^{7}$ Patients gradually become aware of a painless blur in the center of their reading vision, which continues to progress slowly. The insidious onset and slow progression of the symptoms often delay early detection; hence, there is a consequent delay in its management. Central scotoma is the most common visual field defect and commonly associated with dyschromatopsia. ${ }^{8,9}$ Our patient had nuchal rigidity along with vision loss within a week of starting therapy. TBM was suspected and confirmed on CSF analysis. CT brain showed leptomeningeal enhancement and later at 6 weeks follow-up MRI brain revealed enhancement of optic chiasm with leptomeninges. Hence the possibility of ethambutol toxicity was ruled out.

Recurrence or appearance of fresh symptoms and physical and radiological signs in a patient who had previously shown improvement with appropriate ATT is called as paradoxical reaction. Paradoxical reactions has been reported as early as 2 weeks and as late as 18 months after the initiation of ATT. ${ }^{10}$ Paradoxical reactions occur due to complex interplay between host's immune response. There is enhanced delayed-type hypersensitivity leading to activation and accumulation of lymphocytes and macrophages at the site of bacterial deposition or toxin production when bacilli die. ${ }^{10}$ Paradoxical reaction mainly reponds to steroids. ${ }^{11}$ Our patient developed symptoms within a week of procurement of ATT which is highly unlikely due to paradoxical reaction because it is a delayed-type hypersensitivity which appears between 2 weeks and 18 months of starting therapy. Paradoxical reaction responds well to steroids and our patient was continuously on steroid therapy during the course. Hence, the possibility of paradoxical reaction was ruled out.

The enigma was solved after ruling out the above-mentioned possibilities and the diagnosis of TBM was made with rare involvement of cranial nerves especially primary involvement of $\mathrm{CN} 2$ leading to primary optic atrophy.

Similar to Pott's spine, diagnosis of TBM is challenging and needs high index of suspicion. Elevated ESR and positive tuberculin test are suggestive but not confirmatory. Cerebrospinal fluid analysis is done but can be nondiagnostic. The "gold standard" for diagnosis should ideally be isolation and culture of tuberculous bacilli. However, culture methods are slow and insensitive especially in cases of paucibacillary skeletal lesions. There is currently no single diagnostic method that can detect all tuberculosis types and cases.

Prognosis depends on the timing of treatment. Even short delay in diagnosis and hence treatment leads to serious complications as was seen in our case. The rapid and accurate diagnosis of symptomatic patients is the cornerstone of global strategies for TB control. For countries sinking under TB's load, inadequate access to good diagnostics at all health service levels leaves many patients undiagnosed. So it raises a caution for clinicians that a close and keen clinical evaluation is mandatory with high suspicion of TB. 
Empiric therapy is recommended based on clinical suspicion and suggestive laboratory findings. Combination chemotherapy is recommended for Pott's spine and TBM. The standard use of triple drug regimen of isoniazid, rifampicin, and pyrazinamide for 12 months is recommended but on behalf of recent increase in drug resistant organisms use of fourth or even fifth anti-TB drugs consisting of ethambutol and/or streptomycin has been suggested. Adjuvant corticosteroid treatment (dexamethasone or prednisolone) should be given at least for one month in severe cases of CNS TB. Role of surgery for Pott's spine yet remains controversial. Some have advocated surgery for every infected site; however, because of effectiveness of chemotherapy in even those with mild neurological deficits, surgery is now mainly reserved for significant or progressive neurological deficit and deformity correction. $^{2}$

\section{Conclusion}

This case reveals one of the rarest complications of Pott's spine that later developed as TBM and finally presented as primary optic atrophy with involvement of third and sixth cranial nerves. It also signifies the pivotal role of early clinical suspicion for tuberculosis and its importance in preventing complications associated with this disease, because the main loss to the patient was her vision due to delay in diagnosis.

\section{Authors' Contributions}

All authors were involved in clinical assessment, management of the patient, and preparation of manuscript. The manuscript has been read and approved by all the authors and each author believes that the manuscript represents honest work.

\section{Funding}

This research did not receive any specific grant from funding agencies in the public, commercial, or not-for-profit sectors.

\section{Conflict of Interest}

None declared.

\section{References}

1 Gautam VKS, Khurana S, Singh R. Diagnostic and therapeutic challenges in the surgical management of CNS Tuberculosis. Int J Med Health Sci 2013;2:161-169

2 Park P, Lewandrowski K, McLain R. Pott's disease associated with tuberculous meningitis causing blindness. Int J Spine Surg 2006;3(2)

3 Nussbaum ES, Rockswold GL, Bergman TA, Erickson DL, Seljeskog EL. Spinal tuberculosis: a diagnostic and management challenge. J Neurosurg 1995;83(2):243-247

4 Leonard JM, Des Prez RM. Tuberculous meningitis. Infect Dis Clin North Am 1990;4(4):769-787

5 Gill B, Heavner JE. Postoperative visual loss associated with spine surgery. Eur Spine J 2006;15(4):479-484

6 Citron KM, Thomas GO. Ocular toxicity from ethambutol. Thorax 1986;41(10):737-739

7 Schild HS, Fox BC. Rapid-onset reversible ocular toxicity from ethambutol therapy. Am J Med 1991;90(3):404-406

8 Melamud A, Kosmorsky GS, Lee MS. Ocular ethambutol toxicity. Mayo Clin Proc 2003;78(11):1409-1411

9 Trusiewicz D. Farnsworth 100-hue test in diagnosis of ethambutol-induced damage to optic nerve. Ophthalmologica 1975;171(6):425-431

10 Gupta M, Bajaj BK, Khwaja G. Paradoxical response in patients with CNS tuberculosis. J Assoc Physicians India 2003;51:257-260

11 Monga PK, Dhaliwal U. Paradoxical reaction in tubercular meningitis resulting in involvement of optic radiation. Indian J Ophthalmol 2009;57(2):139-141 